\title{
A DISTRIBUTED POPULATION OF LOW-MASS PRE-MAIN-SEQUENCE STARS NEAR THE TAURUS MOLECULAR CLOUDS
}

\author{
Catherine L. Slesnick, ${ }^{1}$ John M. Carpenter, ${ }^{1}$ Lynne A. Hillenbrand, ${ }^{1}$ and Eric E. Mamajek ${ }^{2}$ \\ Received 2006 August 3; accepted 2006 August 31
}

\begin{abstract}
We present a drift-scan survey covering a $\sim 5^{\circ} \times 50^{\circ}$ region toward the southern portion of the Taurus-Auriga molecular cloud. Data taken in the $B, R, I$ filters with the Quest-2 camera on the Palomar 48 inch $(1.2 \mathrm{~m})$ Samuel Oschin Telescope were combined with Two Micron All Sky Survey near-infrared photometry to select candidate young stars. Follow-up optical spectroscopy of 190 candidates led to the identification of 42 new low-mass pre-mainsequence stars with spectral types M4-M8, of which approximately half exhibit surface gravity signatures similar to known Taurus stars, while the other half exhibit surface gravity signatures similar to members of the somewhat older Upper Scorpius, TW Hya, and $\beta$ Pic associations. The pre-main-sequence stars are spread over $\sim 35^{\circ}$, and many are located well outside of previously explored regions. From assessment of the spatial and proper-motion distributions, we argue that the new pre-main-sequence stars identified far from the clouds cannot have originated from the vicinity of the 1-2 Myr old subclusters that contain the bulk of the identified Taurus members but instead represent a newly identified area of recent star formation near the clouds.
\end{abstract}

Key words: open clusters and associations: individual (Taurus) — stars: low-mass, brown dwarfs — stars: pre-main-sequence

Online material: machine-readable tables

\section{INTRODUCTION}

The Taurus-Auriga molecular cloud complex has for decades been considered the quintessential example of low-density, isolated star formation. This fact, along with the proximity of Taurus $(140 \mathrm{pc})$ and its position in the northern hemisphere, has caused Taurus to be one of the most often studied star-forming regions. Several authors (e.g., Briceño et al. 1999, 2002; Luhman 2000, 2003, 2004; Guieu et al. 2006) have used optical/near-infrared/ $\mathrm{X}$-ray imaging to identify young star candidates within the clouds. Follow-up optical spectroscopy of photometrically selected candidates can distinguish members of Taurus from foreground or background field interlopers. These studies found that the Taurus population is clustered into several loose aggregates (Gomez et al. $1993)$ and is predominantly very young ( 1-2 Myr; e.g., Briceño et al. 1998; Hartmann 2000). Thus far, no conclusive evidence has been established for a widespread population of older stars within or near the cloud (Briceño et al. 1999).

The phenomenon of short-timescale (1-2 Myr) clustered star formation is not unique to Taurus but has been found in almost all other nearby young associations (e.g., Carpenter 2000; Palla $\&$ Stahler 2000). The large numbers of very young stars and the apparent lack of more evolved (5-10 Myr old) objects in starforming regions contrasts with the ages of a few tens of megayears (e.g., Blitz \& Shu 1980) inferred for molecular clouds. Either star formation takes place for only a small fraction of the cloud lifetime, or molecular clouds themselves live only a few megayears (e.g., Hartmann et al. 2001). This problem has been discussed in the literature for almost three decades and is commonly known as the "Post T Tauri Star Problem" (Herbig 1978).

An alternative explanation for the apparent lack of older stars in molecular clouds is that such objects have been missed in previous surveys. Limitations in telescope time and instrument

\footnotetext{
${ }^{1}$ Department of Astronomy, California Institute of Technology, Pasadena, CA, USA; cls@astro.caltech.edu,jmc@astro.caltech.edu, lah@astro.caltech.edu.

2 Harvard-Smithsonian Center for Astrophysics, Cambridge, MA, USA; emamajek@cfa.harvard.edu.
}

fields of view have constrained most previous deep imaging surveys in Taurus (e.g., Briceño et al. 1999; Luhman 2000) to small areas focused on subclusters (each $\sim 1 \mathrm{pc}$ wide) that contain most of the known young members. Assuming a mean velocity dispersion of $\sim 2 \mathrm{~km} \mathrm{~s}^{-1}$ (Hartmann et al. 1986), a putative population of 5-10 Myr old stars in Taurus could travel up to $\sim 20 \mathrm{pc}\left(8^{\circ}\right)$ away from its birth site.

Several studies (e.g., Neuhäuser et al. 1997; Wichmann et al. 1996) have attempted to use the ROSAT All Sky Survey (RASS) with spectroscopic follow-up to identify G to mid-K type post T Tauri stars (PTTSs; stars with ages $\sim 3-10 \mathrm{Myr}$ ) far from the current Taurus members. These observations revealed a distributed population of lithium-rich stars (indicating that they are younger than $\sim 100 \mathrm{Myr}$ ) that are widely dispersed across the cloud and beyond. However, because both the decay of X-ray emission and the depletion of lithium occur slowly for G-type stars with ages $<100 \mathrm{Myr}$, these data alone cannot discriminate whether the RASS-selected stars represent a $1 \mathrm{Myr}$ old or $10 \mathrm{Myr}$ old Taurus population, or a 100 Myr old population that originated elsewhere. Consequently, the origin of these stars and their relation to Taurus is still controversial (e.g., Briceño et al. 1997). The largest optical/near-infrared imaging survey to date that searched for Taurus members is that of Luhman (2006), who used a combination of USNO and Two Micron All Sky Survey (2MASS) magnitudes to find young brown dwarfs within a $15 \times 15 \mathrm{deg}^{2}$ region centered on the known $1 \mathrm{Myr}$ old subclusters. The survey was aimed specifically at identifying young brown dwarfs with colors and magnitudes similar to known $\sim 1$ Myr old substellar Taurus members and was not targeted at finding older objects or comparably aged low-mass stars.

We have completed a new optical $U, B, R, I$ imaging survey of $\sim 250 \mathrm{deg}^{2}$ near the Taurus molecular clouds. The specific survey area was chosen to include both well-known subclusters of stars and regions beyond previously studied parts of the cloud. The current work is a companion survey to a photometric and spectroscopic study we have carried out in the Upper Scorpius (USco) star-forming region (Slesnick et al. 2006, hereafter SCH06), 
where we identified 43 new brown dwarfs and low-mass stars. Our primary goal in Taurus is to search for and characterize a possible distributed population of pre-main-sequence (PMS) stars within and surrounding the clouds. We combine the Taurus photometric data with 2 MASS $J, H, K_{s}$ photometry to select candidate young stars $(\S 2)$. In $\S 3$ we present newly obtained spectral data for 190 of these candidates, from which we identify a population of 42 low-mass PMS objects. We assess the spatial and proper-motion distributions of the new population in $\S 4$ and discuss why these objects may have been missed in previous surveys.

\section{QUEST IMAGING OBSERVATIONS}

\subsection{Photometric Monitoring and Data Processing}

$U, B, R, I$ observations were obtained with the Quest-2 Camera (Rabinowitz et al. 2003) on the 48 inch (1.2 m) Samuel Oschin Schmidt Telescope at Palomar Observatory. The Quest-2 camera is a large-area mosaic consisting of $112 \mathrm{CCDs}$ arranged in four columns of 28 detectors. The camera covers a $3.6^{\circ} \times 4.6^{\circ}$ field of view and, taking into account gaps between columns and chips, the instantaneous on-sky coverage is $9.4 \mathrm{deg}^{2}$. Each of the four columns views the sky through a separate filter (Johnson $U, B, R$, or $I$ in this case, although our data are calibrated to the Sloan photometric system; see below). We operated the camera in driftscan mode, where the final data product is a strip of uniform width in declination and time-dependent length in right ascension that has been imaged in four filters.

One strip, covering the right ascension range $40^{\circ} \lesssim \alpha \lesssim 90^{\circ}$ and spanning $4.6^{\circ}$ in declination, centered on $\delta=22.5^{\circ}$, was observed twice per night on the nights of 2003 November 27-30 and 2003 December 4-5 for a total of 12 scans of the same portion of the sky. This spatial area includes the young regions of L1536 and L1529, as well as the Pleiades open cluster $\left(\alpha=57^{\circ}\right.$, $\delta=24^{\circ}$ ). The CCDs are less sensitive in the $U$ band than anticipated, and few sources were detected. We therefore exclude the $U$-band data from the remainder of this discussion. Fourteen of the $84 B, R, I$ CCDs have failed since installation due to bad connections or faulty chips, rendering our spatial coverage within the survey region nonuniform.

Data reduction, source extraction, and photometry were carried out using the Yale reduction pipeline as outlined in SCH06. After bias subtraction, dark correction, and flat-fielding (see SCH06 for more details), the pipeline performs aperture photometry for all stars through an aperture of half-width 3.5 pixels. We have matched detections within a $0.8^{\prime \prime}$ radius from the 12 different scans of the survey region. For each source we averaged coordinates from individual scans. The typical astrometric rms deviation about the mean is $\sim 0.13^{\prime \prime}$ for stars detected in at least half of the scans. The average offset between Quest-2 and 2MASS coordinates (see $\S 2.2)$ is $+0.2^{\prime \prime}$ in right ascension and $+0.04^{\prime \prime}$ in declination.

To account for nonlinearity and pixel-to-pixel variations within a chip and zero-point and color variations between chips, we matched a subset of data $(\sim 600,000$ stars $)$ taken by the Quest- 2 collaboration (Rabinowitz et al. 2003) in a different part of the sky $\left(-2.5^{\circ} \lesssim \delta \lesssim 2.5^{\circ}, 120^{\circ} \lesssim \alpha \lesssim 240^{\circ}\right)$ to the Sloan Digital Sky Survey (York et al. 2000). For each of the Quest-2 CCDs we computed a conversion from Quest-2 to Sloan magnitudes in the form of

$$
\begin{aligned}
r & =a_{R}+b_{R} R_{\text {Quest }}+c_{R} \text { Row }+d_{R}(R-I)_{\text {Quest }}, \\
i & =a_{I}+b_{I} I_{\text {Quest }}+c_{I} \text { Row }+d_{I}(R-I)_{\text {Quest }}, \\
b & =a_{B}+b_{B} B_{\text {Quest }}+c_{B} \text { Row }+d_{B}(B-R)_{\text {Quest }},
\end{aligned}
$$

where $a_{X}, b_{X}, c_{X}$, and $d_{X}$ are constants and "Row" refers to a row of pixels (perpendicular to the drift scan direction) on the CCD.
Only stars with both Sloan and Quest-2 photometric uncertainties $<0.1$ mag and instrumental Quest-2 magnitudes between $11.5<R, I<19$ and $14<B<21$ were used to derive the calibrations. Constants were computed in discrete bins of $0.75 \mathrm{mag}$, 50 pixels, and $0.2 \mathrm{mag}$ in color and determined in an iterative manner for each equation above until each constant changed by $<0.0005$ mag for at least three iterations. We applied the derived calibrations to the Taurus scans by linearly interpolating between values in each parameter.

The Taurus data were taken primarily under nonphotometric conditions. However, because the data consists of repeated scans, self-calibration is possible. To account for variable conditions during a night or from night to night, we applied a photometric offset as a function of right ascension to each scan. For a given $\mathrm{CCD}$, the Yale reduction pipeline divides a drift scan into discrete frames 2048 pixels in length. Due to edge effects, along with the fact that some of the data were taken through thick clouds ( $\geq 2$ mag of extinction), most sources were not detected in all 12 scans. The "best" (i.e., most photometric) scan, taken on 2003 November 27, was used to define the photometric reference system. We selected a subset of $\sim 100,000$ stars from the source catalog that were detected in at least 6 of 12 scans (including the reference scan) and had no neighbors within $5^{\prime \prime}$. For each such star we computed the difference between the reference magnitude and the magnitude measured in an individual scan.

For every chip and scan we created a catalog of photometric offsets by stepping through in right ascension every five local calibrator stars (typically spanning $\sim 0.5^{\circ}$ ) and calculating a median offset value between an individual scan and the reference scan. Typical values were $0.18 \mathrm{mag}$ in $r, 0.15 \mathrm{mag}$ in $i$, and $0.23 \mathrm{mag}$ in $b$. We applied these offsets to the entire data set as a function of right ascension, scan, and CCD by linearly interpolating between values in right ascension. Offsets as a function of declination were also computed. However, on examination we found no systematic structure in the declination offsets and thus only the right ascension offsets were necessary.

The photometric precision can be assessed from the repeatability of observations for individual stars. For each source we computed an uncertainty-weighted average and the rms deviation of individual calibrated measurements. The rms deviation can be affected by many factors, including photometric noise, uncertainties in the derived Quest-2-to-Sloan calibrations or scan-to-scan weather calibrations, and the intrinsic photometric variability of the star. Because the 112 CCDs within the mosaic are of varying quality, defining "typical" rms deviation is meaningful only on a chip-by-chip basis. From analysis of rms values as a function of magnitude for all CCDs, average rms values for most chips range from 0.03 to 0.1 mag for stars brighter than $r, i \sim 20$ and $b \sim 21$ and are as high as 0.2 mag for a few CCDs. Scatter increases toward fainter magnitudes as expected (see Fig. 2 in SCH06).

The accuracy of the absolute photometry is harder to quantify. While we have accounted for relative extinction due to weather within our data set, we are not able to account for zero-point shifts between the Taurus drift scans and the scans used to derive the Quest-2-to-Sloan calibrations. Despite this fact, comparison of the average $r-i$ color for the calibrated Quest-2 photometry agrees to $\sim 0.01 \mathrm{mag}$ with the average $r-i$ color of the $\sim 600,000$ stars from Sloan used to derive the calibrations. Therefore, while we cannot claim the photometry is on a standard Sloan $b, r, i$ system, it should be fairly closely aligned with Sloan. Nevertheless, we select candidate PMS stars based on a combination of relative Quest-2 optical colors and absolute 2MASS near-infrared colors (see $\S 2.2$ ). The final source catalog contains $\sim 2.2$ million 


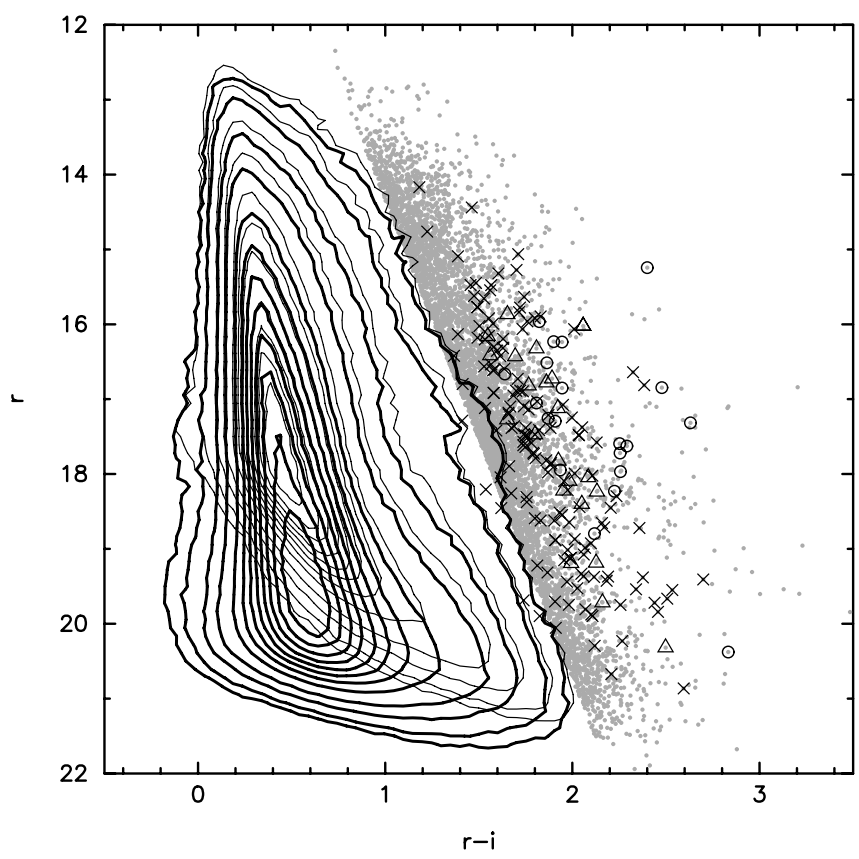

FIG. 1.-Optical CMD of all $\sim 2.2$ million Quest-2 sources with $r$ and $i$ detections (thick contours) and those with 2MASS counterparts (thin contours). Contours represent data at $90 \%-10 \%, 5 \%, 2 \%$, and $1 \%$ of the peak level. Objects redward of a linear approximation of the $1 \%$ contour are shown as discrete gray dots. Objects for which we have spectral data are shown as large symbols: spectroscopically confirmed young stars are shown as circles, intermediate-age stars as triangles, and dwarf stars as crosses.

sources within the $\sim 250 \mathrm{deg}^{2}$ survey region with both $r$ - and $i$-band photometry.

\subsection{Candidate Pre-Main-Sequence Selection}

Our goal is to use the photometry to isolate PMS stars both within and surrounding the Taurus clouds from the field-star population that dominates the source catalog. Nearby young stars still undergoing contraction are systematically more luminous than their main-sequence counterparts and will therefore occupy a sequence in an optical color-magnitude diagram (CMD) that is correspondingly brighter than the sequence occupied by most of the field stars. Due to challenges in calibrating the photometric data to a standard system, we were reluctant to choose candidates based on colors and magnitudes that correspond to particular isochronal ages. For the initial spectroscopic observations, we considered as candidate PMS stars all sources redward of a linear approximation of the $1 \%$ data contour in an $r, r-i$ CMD (see Fig. 1).

In addition to optical colors, the infrared colors and magnitudes of potential PMS candidates were also considered. We matched the entire source catalog to 2MASS (Skrutskie et al. 2006) and excluded from further consideration $\sim 1.2$ million sources that did not have a 2MASS counterpart. In Figure 1, optical CMDs for all Quest-2 sources and for sources with a 2MASS counterpart are shown. As discussed in SCH06, requiring a 2MASS detection biases the list of potential PMS candidates against faint blue sources but does not exclude red objects bright enough to be observed spectroscopically at Palomar $(r \lesssim 20.5)$. The position of each star on a near-infrared color-color diagram was examined, and any star with $J-H, H-K_{s}$ colors consistent with those of background giants $\left[(J-H)>0.6\left(H-K_{s}\right)+0.6\right.$ or $(J-H)>$ $\left.1.69\left(H-K_{s}\right)+0.29\right]$ was excluded. We additionally considered $r-K_{s}$ colors and adopted the selection criterion $r<2.57(r-$ $\left.K_{s}-3\right)+13$, as outlined in SCH06. After all selection criteria

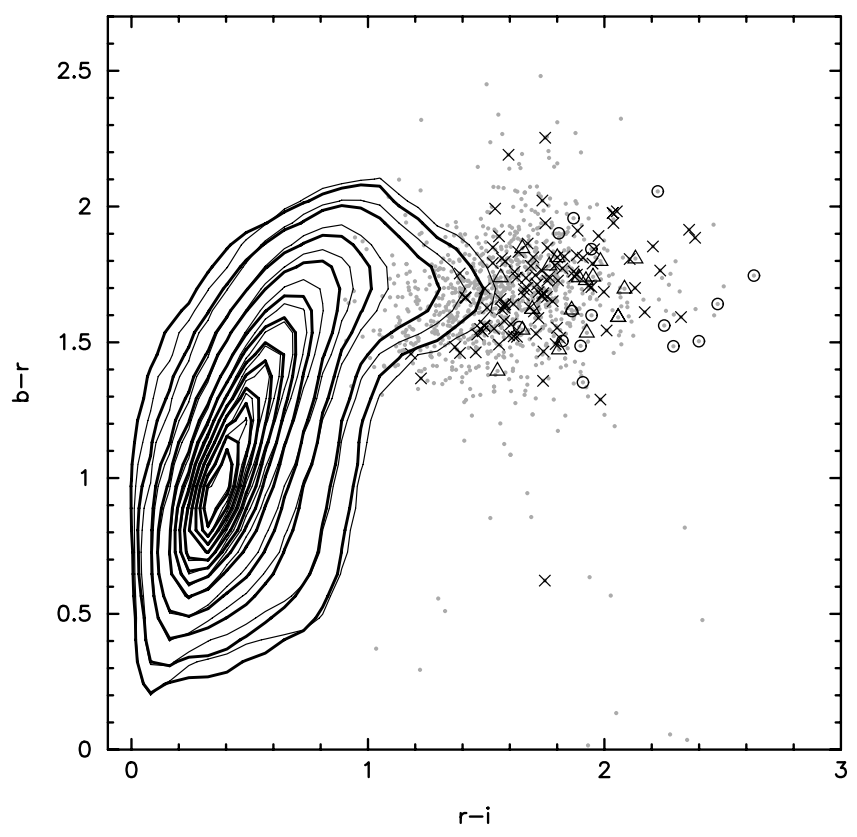

FIG. 2.-Optical color-color diagram of the $\sim 1$ million Quest-2 sources with $r, i$, and $b$ data (thick contours) and of those with 2MASS counterparts (thin contours) represented at $90 \%-10 \%, 5 \%, 2 \%$, and $1 \%$ of the peak level. Objects that meet all of the $\left(r i J H K_{s}\right)$ selection criteria to be candidate PMS stars and have $b$ data are shown as discrete gray dots. Large symbols are as in Fig. 1.

were applied, the final candidate list contains $\approx 1800$ stars for spectroscopic follow-up.

Although $b$-band data was not used in candidate PMS selection, $\approx 60 \%$ of our candidates and 136 out of 190 objects observed spectroscopically (see $\S 3$ ) have a $b$-band detection. Figure 2 shows an $(r-i)$ versus $(b-r)$ color-color diagram for all stars with $r, i, b$ detections and for stars that additionally have 2MASS detections. Data with $b$-band detections meeting the selection criteria outlined above are shown as discrete dots. In principle, $b$ band (and $u$-band) photometry could be used to select candidate young stars based on a blue excess attributed to accretion (e.g., Rebull et al. 2000), although we have not implemented any such criteria in the present work.

\section{OPTICAL SPECTROSCOPY}

Moderate-resolution spectra of 190 candidate PMS objects (chosen from the $\sim 1800$ candidates discussed in $\S 2.2$ ) were taken with the Double Spectrograph on the Palomar 200 inch (5 m) Hale Telescope on the nights of 2004 December 9-11 and 2005 November 23-27. Candidates were prioritized by $r-K_{s}$ color, with redder candidates observed first, and targets were selected primarily from the magnitude range $15<r<19$. The spatial distribution of observed stars is discussed in $\S$ 4.1. Data were taken with the red side of the spectrograph through a $2^{\prime \prime}$ slit using a $5500 \AA$ dichroic and a 316 lines $\mathrm{mm}^{-1}$ grating blazed at $7500 \AA$. This setup produced wavelength coverage from 6300 to $8825 \AA$ at a resolution of $R \sim 1250$. Typical exposure times were 300$900 \mathrm{~s}$ and up to $1800 \mathrm{~s}$ for the faintest targets $(r \sim 21)$. Spectrophotometric standard stars (Massey et al. 1988) were observed throughout each night for flux calibration. All sources were processed, extracted, and calibrated using standard IRAF tasks.

In addition to our program targets we have also observed a range of spectral main-sequence standards (K5-L3), giant standards (K7-M9), and previously identified Taurus objects (K3M7.25; Briceño et al. 2002; Luhman 2004). We observed several members of the Hyades ( 650 Myr; Lebreton et al. 2001), 

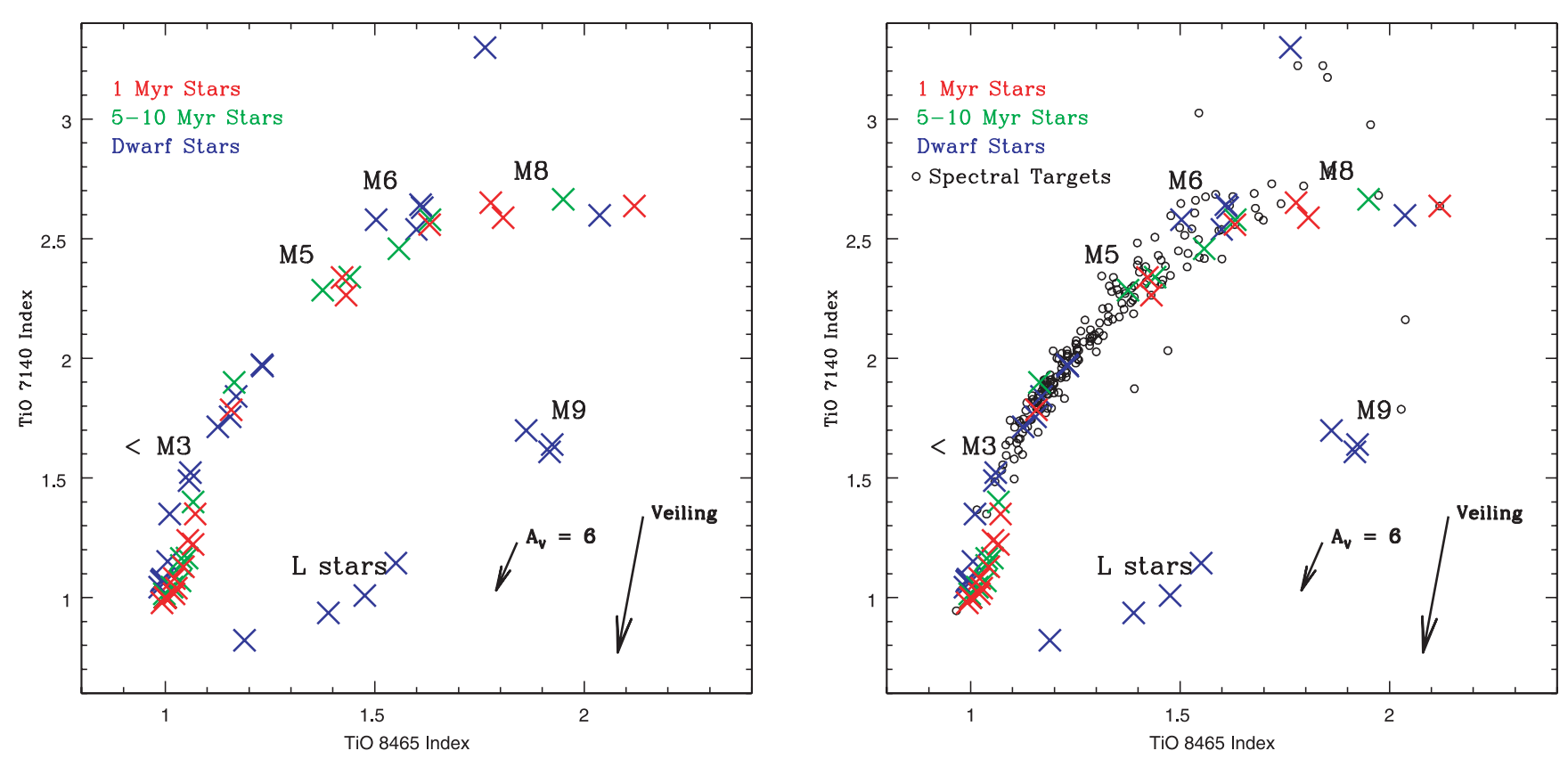

FIG. 3.- Temperature-sensitive TiO-7140 vs. TiO-8465 indices. Blue crosses represent measured indices for field dwarfs and members of the Hyades ( 650 Myr), Pleiades ( $\sim 115 \mathrm{Myr})$, and AB Dor ( $\sim 75-150 \mathrm{Myr})$ associations. Green crosses show measured indices for intermediate-age spectral standards from $\beta$ Pic $(\sim 11 \mathrm{Myr})$, TW Hya ( $\sim 8 \mathrm{Myr}$ ), and USco ( $\sim 5 \mathrm{Myr})$. Red crosses show measured indices for young Taurus members ( $\sim 1-2 \mathrm{Myr})$. In the right panel, black symbols indicate measured indices for Quest-2 PMS candidates. The effects of extinction and veiling are shown as vectors (see text). This diagram is useful for classifying stars with spectral types M3-L3.

Pleiades ( 115 Myr; Basri et al. 1996), AB Dor ( 75-150 Myr; Luhman et al. 2005), $\beta$ Pic ( 11 Myr; Ortega, et al. 2004), and TW Hya ( $\sim 8$ Myr; de la Reza et al. 2006) associations. In the SCH06 survey we observed $>50$ members of the $\sim 5$ Myr USco association. Together, these observations provide a large set of late spectral type $(\mathrm{K}-\mathrm{M})$ standards spanning a broad range of age (surface gravity).

\subsection{Spectral Analysis}

A detailed discussion of our classification methods is given in SCH06, and thus only a summary is provided here. We use the strength of titanium oxide ( $\mathrm{TiO}$ ) absorption, which increases from mid-K through $\sim \mathrm{M} 7$ spectral types (see Fig. 8 of SCH06), as the primary spectral type diagnostic. We have adopted from the literature two spectral indices that measure the strength of TiO features: TiO-7140 $\left(F_{\lambda 7035} / F_{\lambda 7140}\right.$ with bandwidths of $50 \AA$; Wilking et al. 2005) and TiO-8465 $\left(F_{\lambda 8415} / F_{\lambda 8465}\right.$ with bandwidths of $20 \AA$; SCH06). The left side of Figure 3 shows a plot of TiO-8465 versus TiO-7140 for observed spectral standards. This diagram is useful for classifying stars with spectral types M3-L3. No significant age dependence exists between the measured $\mathrm{TiO}$ indices of older stars (ages $>75 \mathrm{Myr}$; blue crosses) and young (ages 1-2 Myr; red crosses) or intermediate-age (ages 5-10 Myr; green crosses) PMS standard stars.

Measured indices for program sources are shown in the right side of Figure 3. The measurements predominantly follow the locus determined by the spectral standards. Two outliers sit below the primary sequence of data points. Both objects are confirmed to be young stars (see below) with strong $\mathrm{H} \alpha$ emission, and we attribute their position in Figure 3 to a small amount of veiling present in their spectra (see $\S 3.2$ ). Spectral types were first estimated from quantitative analysis of the measured $\mathrm{TiO}$ indices. More weight was given to the TiO-8465 index, which is the less sensitive of the TiO indices to the effects of reddening and veiling (\$3.2). Final spectral types were confirmed after visual inspection of each spectrum in comparison to spectral standards.
In addition to determining spectral types, it was also necessary to determine which of the candidates are bona fide PMS stars. Because young stars are still undergoing contraction to the main sequence, they have systematically lower surface gravity than older main-sequence stars. We therefore use surface gravity to roughly define stellar age. Several diagnostics of surface gravity exist in this wavelength regime, which can be assessed in lowand moderate-resolution spectra. The most prominent gravitysensitive features in the Palomar spectra are the K I (7665 and $7669 \AA)$ and $\mathrm{Na}$ I (8183 and $8195 \AA$ ) absorption doublets.

We assess surface gravity quantitatively by using the gravitysensitive Na-8189 index $\left(F_{\lambda 8189} / F_{\lambda 8150}\right.$ with bandwidths of $30 \AA$; $\mathrm{SCH06)}$ to measure the strength of the Na I doublet. Figure 4 shows a plot of the temperature-sensitive TiO-8465 index as a function of the gravity-sensitive Na-8189 index for all stars earlier than M9. On the left is shown measured indices for spectral standards. Colors are as in Figure 3, with the addition of cyan crosses for giant stars. The right side of Figure 4 shows measured indices for objects that are classified as having low (circles) or intermediate (triangles) gravity and candidates spectroscopically determined to be field dwarfs (black crosses; see below).

One difficulty in interpreting Figure 4 directly is that the Na-8189 index is contaminated by telluric features. This point was not discussed in $\mathrm{SCH} 06$ because both the candidates and USco spectral standards were taken at similar air masses, and thus this effect did not influence the spectral classifications. However, the Taurus spectral data were taken at systematically lower air masses than many of the spectral standards. As a result, a program star with intermediate-gravity signatures observed at low air mass will have a lower Na-8189 index than an intermediate-gravity standard of the same spectral type observed at high air mass. This effect causes a discrepancy between the positions of the green crosses and the triangles in Figure 4.

Figure 5 shows a section of the spectra that highlights the $\mathrm{Na}$ I ( 8183 and $8195 \AA$ ) feature for dwarf and intermediate- and lowgravity stars of the same spectral type. Both GJ 866 and USco 

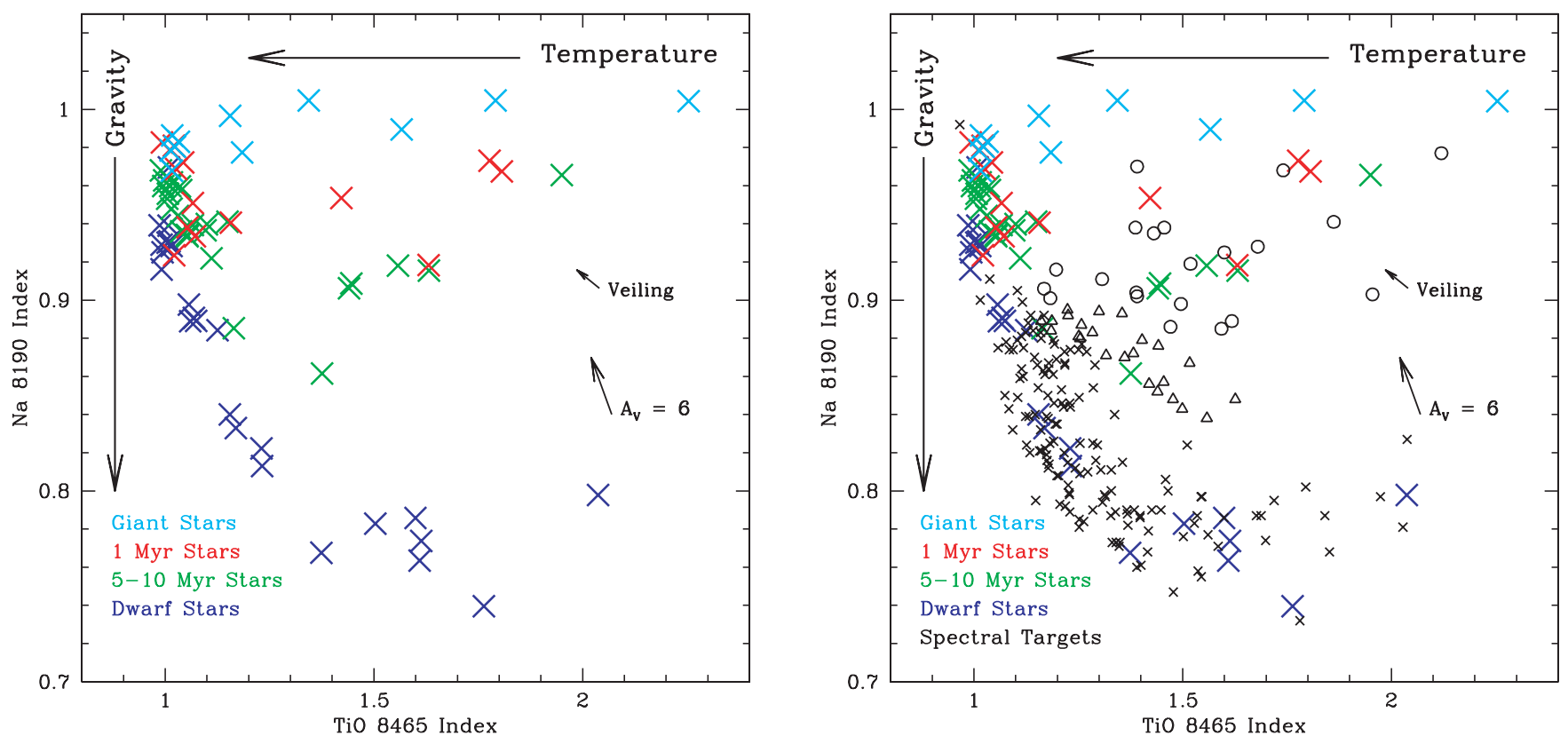

FIG. 4.- TiO-8465 index vs. the gravity-sensitive Na-8189 index. Symbols are as in Fig. 3 with the addition of cyan crosses that represent measured indices for giant standard stars. Objects with higher surface gravity have more Na I absorption present in their spectra and thus a smaller Na-8189 index. In the right panel, black symbols representing program candidates are divided into three groups based on inferred surface gravity: circles represent objects with surface gravity lower (i.e., younger in age) than the USco association, triangles indicate objects with intermediate surface gravity comparable to the USco, TW Hya, and $\beta$ Pic associations, and black crosses indicate field stars. As in Fig. 3, the effects of veiling and extinction are shown as vectors (see text).

CTIO 53 (the dwarf and intermediate-gravity stars) were observed at high air mass, whereas MHO 7 (the low-gravity Taurus member) was observed at low air mass. Telluric absorption (8161$8282 \AA$ ) seen in the spectra of GJ 866 and USco CTIO 53 affects both the continuum band and the $\mathrm{Na}$ I band, causing systematically high measurements of the Na-8189 index. However, the three spectra can be clearly distinguished through visual inspection of the Na I line strengths. Thus, we used the quantitative indices as a rough guide only, and all final gravity classification was done by eye. All objects with surface gravity features weaker than those of the intermediate-age standards are considered by us to have low gravity; objects with gravity features similar to those exhibited by the intermediate-age standards are considered to have intermediate gravity. Based on this classification scheme, we identify

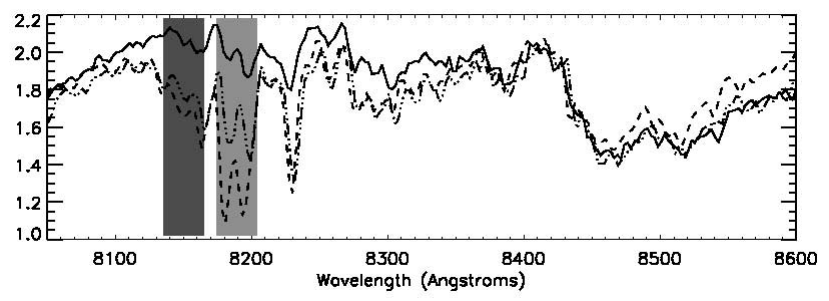

FIG. 5. - Section of the optical spectrum highlighting the surface-gravity-sensitive $\mathrm{Na}$ I doublet (8183 and $8195 \AA$ ). The dashed spectrum represents an M5 V star (GJ 866; Kirkpatrick et al. 1991), the dot-dashed spectrum represents an M5 USco member (USco CTIO 53; Ardila et al. 2000), and the solid spectrum represents an M5 Taurus member (MHO 7; Briceño et al. 1998). Light and dark shaded regions show the respective locations of the $\mathrm{Na}$ I and the continuum bands used in constructing the Na-8189 index. All spectra have been normalized at $8410 \AA$ near the temperature-sensitive TiO ( $8465 \AA$ ) molecular absorption band. Both GJ 866 and USco CTIO 53 were observed at high air mass, and telluric absorption (8161$8282 \AA$ ) affects both the continuum band and the Na I band, causing systematically high measurements of the Na-8189 index. However, gravity signatures in the three spectra can be distinguished clearly through visual inspection of the line strengths.
42 new PMS stars (see Table 1). Of these, 19 exhibit low surface gravity features, and the remaining 23 exhibit intermediate surface gravity features. A detailed discussion of both populations is given in $\S 4$.

\subsection{Extinction and Veiling}

Although most of the known low-mass Taurus members have low extinction $\left(A_{V} \sim 1\right.$; e.g., Kenyon et al. 1994), the quantitative indices used in classification are affected by interstellar reddening, which we must account for in the classification process. To assess any potential effect, we artificially reddened all spectral standards by $A_{V}=6 \mathrm{mag}$, the maximum extinction within our survey region as inferred from large-beam dust maps (Schlegel et al. 1998). We then remeasured all classification indices. Results of this experiment for an M5 star are shown as vectors on Figures 3 and 4. Reddening at this level is not sufficient to shift the indices by more than $\approx 0.5$ subclasses in temperature and can be easily identified on visual assessment of the overall spectral slope.

We must also consider the effects of veiling, either due to excess emission from an accretion shock or due to thermal emission from dust grains in a circumstellar disk. In SCH06 we explored possible biases introduced by these processes on our spectral classification by adding to the spectra hot or cold blackbody emission of constant temperature or continuum excess of constant flux. $\mathrm{SCH} 06$ found that veiling due to thermal emission from dust grains in a disk does not significantly affect the spectral classification indices used here. Veiling from a hot accretion shock, however, does systematically decrease the TiO-7140 index. The maximum shifts produced from these experiments are shown as vectors in Figures 3 and 4. We believe this effect to be the cause of the measured indices for SCH J0429595+2433080 and SCH J0518028+2327126 lying beneath the primary sequence of points. In addition to this evidence for veiling, both stars are confirmed to have low gravity and strong $\mathrm{H} \alpha$ emission consistent with young, 
TABLE 1

Measured Quantities for New PMS Stars

\begin{tabular}{|c|c|c|c|c|c|c|c|c|c|c|c|c|}
\hline $\mathrm{ID}^{\mathrm{a}}$ & $b$ & $r$ & $i$ & $J^{\mathrm{b}}$ & $H^{\mathrm{b}}$ & $K_{S}^{\mathrm{b}}$ & TiO-7140 & TiO-8165 & Na-8195 & Sp. Type & $\begin{array}{c}W(\mathrm{H} \alpha) \\
(\AA)\end{array}$ & Age \\
\hline SCH J0325332+2426581.. & 18.0 & 16.4 & 14.7 & 12.34 & 11.72 & 11.47 & 2.02 & 1.30 & 0.89 & M4.5 & -10 & Int \\
\hline SCH J0359099+2009362....................... & 19.8 & 18.0 & 16.1 & 13.47 & 12.89 & 12.53 & 2.32 & 1.42 & 0.85 & M4.75 & -7 & Int \\
\hline 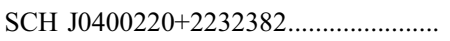 & $\ldots$ & 18.4 & 16.3 & 13.45 & 12.76 & 12.45 & 2.23 & 1.36 & 0.87 & M4.75 & -6 & Int \\
\hline 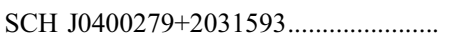 & 20.0 & 18.2 & 16.1 & 13.14 & 12.56 & 12.23 & 2.54 & 1.49 & 0.84 & M5.75 & -16 & Int \\
\hline SCH J0407246+2332554....................... & 18.4 & 16.7 & 14.8 & 12.77 & 12.06 & 11.85 & 1.69 & 1.16 & 0.88 & M4 & -6 & Int \\
\hline SCH J0407350+2237396......................... & 18.6 & 16.8 & 15.0 & 12.16 & 11.60 & 11.25 & 2.38 & 1.51 & 0.86 & M5 & -16 & Int \\
\hline 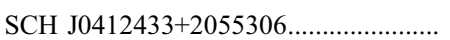 & $\ldots$ & 20.3 & 17.8 & 14.24 & 13.53 & 13.17 & 2.97 & 1.95 & 0.90 & M8 & -15 & Int \\
\hline SCH J0416272+2053093 & 18.6 & 16.8 & 14.9 & 12.05 & 11.47 & 11.11 & 2.26 & 1.43 & 0.93 & M5 & -5 & Young \\
\hline 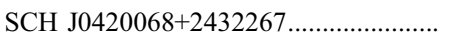 & 17.5 & 16.1 & 14.6 & 12.42 & 11.87 & 11.59 & 1.84 & 1.18 & 0.88 & M4 & $\ldots$ & Int \\
\hline SCH J0420491+2327370....................... & 18.3 & 16.7 & 14.9 & 12.07 & 11.39 & 11.09 & 2.03 & 1.25 & 0.88 & M4.25 & -8 & Int \\
\hline SCH J0426452+2131408....................... & 19.3 & 17.8 & 15.8 & 13.19 & 12.64 & 12.31 & 2.23 & 1.38 & 0.87 & M4.75 & -8 & Int \\
\hline SCH J0427074+2215039....................... & $\ldots$ & 17.7 & 15.4 & 12.27 & 11.64 & 11.29 & 2.64 & 1.74 & 0.96 & M6.75 & -18 & Young \\
\hline SCH J0429595+2433080 …................... & $\ldots$ & 17.9 & 15.7 & 11.68 & 10.53 & 9.81 & 1.87 & 1.39 & 0.97 & M5.5 & -71 & Young \\
\hline SCH J0431191+2335048 ${ }^{\mathrm{c}}$. & $\ldots$ & 20.3 & 17.5 & 13.50 & 12.71 & 12.19 & 2.63 & 2.12 & 0.97 & M8 & -32 & Young \\
\hline 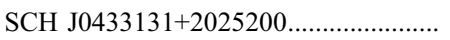 & $\ldots$ & 19.1 & 17.0 & 14.20 & 13.46 & 13.14 & 2.36 & 1.40 & 0.87 & M5 & -12 & Int \\
\hline SCH J0434454+2308035 ........................ & 20.2 & 18.2 & 16.0 & 12.80 & 12.02 & 11.70 & 2.31 & 1.45 & 0.93 & M5.25 & -11 & Young \\
\hline 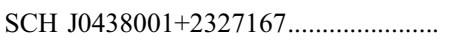 & 19.7 & 18.0 & 15.9 & 13.27 & 12.66 & 12.34 & 2.41 & 1.45 & 0.85 & M5.25 & -15 & Int \\
\hline 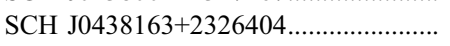 & 17.7 & 16.2 & 14.3 & 11.80 & 11.24 & 10.96 & 2.24 & 1.38 & 0.93 & M4.75 & -7 & Young \\
\hline SCH J0438586+2336352......................... & 18.4 & 16.8 & 14.3 & 11.97 & 11.36 & 11.03 & 2.03 & 1.19 & 0.91 & M4.25 & -15 & Young \\
\hline 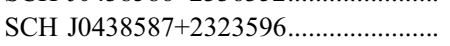 & 19.1 & 17.5 & 15.3 & 12.49 & 11.93 & 11.59 & 2.62 & 1.68 & 0.92 & M6.5 & -14 & Young \\
\hline 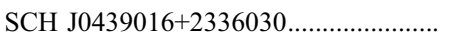 & 16.7 & 15.2 & 12.8 & 11.33 & 10.59 & 10.18 & 2.41 & 1.60 & 0.92 & M6 & -62 & Young \\
\hline SCH J0439064+2334179....................... & 19.0 & 17.3 & 14.6 & 12.09 & 11.53 & 11.19 & 2.78 & 1.86 & 0.94 & M7.5 & -8 & Young \\
\hline SCH J0439410+2304262........ & $\ldots$ & 19.1 & 17.1 & 14.41 & 13.81 & 13.46 & 2.09 & 1.31 & 0.87 & M4.5 & -12 & Int \\
\hline 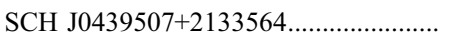 & $\ldots$ & 19.7 & 17.5 & 14.43 & 13.80 & 13.43 & 2.67 & 1.62 & 0.84 & M6 & -8 & Int \\
\hline SCH J0440534+2055473........................ & 18.8 & 17.1 & 15.1 & 12.48 & 11.88 & 11.62 & 2.32 & 1.44 & 0.87 & M5. & -11 & Int \\
\hline 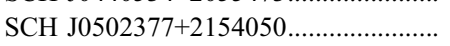 & 19.2 & 17.4 & 15.6 & 13.16 & 12.53 & 12.20 & 1.99 & 1.25 & 0.88 & M4.25 & -10 & Int \\
\hline 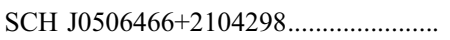 & 18.1 & 16.5 & 14.6 & 12.05 & 11.40 & 11.11 & 2.43 & 1.51 & 0.91 & M5.25 & -14 & Young \\
\hline SCH J0516021+2214530........................ & 17.4 & 15.9 & 14.1 & 11.67 & 11.13 & 10.75 & 2.25 & 1.39 & 0.90 & M5 & -10 & Young \\
\hline 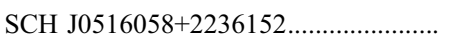 & 19.1 & 17.2 & 15.6 & 13.29 & 12.59 & 12.30 & 1.88 & 1.18 & 0.88 & M4 & -8 & Int \\
\hline 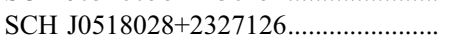 & 18.6 & 17.2 & 15.3 & 12.99 & 12.32 & 11.88 & 2.03 & 1.47 & 0.88 & M5 & -21 & Young \\
\hline 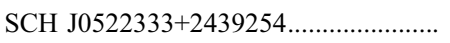 & 18.9 & 17.0 & 15.2 & 12.75 & 12.04 & 11.72 & 2.18 & 1.38 & 0.90 & M4.75 & -7 & Young \\
\hline 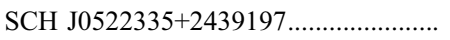 & 19.2 & 17.2 & 15.3 & 12.79 & 12.14 & 11.79 & 2.10 & 1.30 & 0.91 & M4.5 & -8 & Young \\
\hline 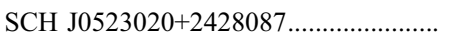 & 18.1 & 16.4 & 14.8 & 12.60 & 11.92 & 11.61 & 1.78 & 1.16 & 0.90 & M4 & -3 & Int \\
\hline SCH J0523500+2435237....................... & $\ldots$ & 18.7 & 16.6 & 13.81 & 13.14 & 12.77 & 2.63 & 1.61 & 0.88 & M6 & -18 & Young \\
\hline 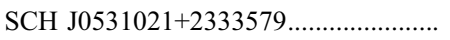 & 17.5 & 16.0 & 14.0 & 12.28 & 11.64 & 11.39 & 1.79 & 1.18 & 0.90 & M4 & -5 & Int \\
\hline 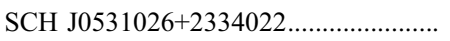 & 17.6 & 16.0 & 13.9 & 12.26 & 11.58 & 11.35 & 1.83 & 1.22 & 0.89 & M4 & -5 & Int \\
\hline SCH J0532021+2423030........................ & 19.9 & 18.2 & 16.2 & 13.69 & 13.06 & 12.80 & 2.50 & 1.44 & 0.85 & M5 & -19 & Int \\
\hline 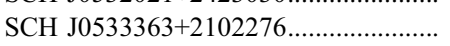 & 17.4 & 15.8 & 14.2 & 11.93 & 11.32 & 11.07 & 2.05 & 1.28 & 0.88 & M4.5 & -7 & Int \\
\hline 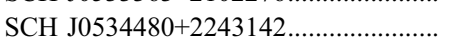 & 18.2 & 16.6 & 15.0 & 12.83 & 12.18 & 11.93 & 1.94 & 1.22 & 0.89 & M4.25 & -6 & Young \\
\hline SCH J0536190+2242428........................ & 17.7 & 16.3 & 14.5 & 12.13 & 11.53 & 11.27 & 2.17 & 1.35 & 0.89 & M4.75 & -12 & Int \\
\hline 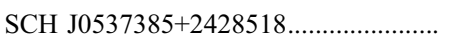 & 17.8 & 16.2 & 14.2 & 11.66 & 11.06 & 10.78 & 2.44 & 1.49 & 0.89 & M5.25 & -14 & Young \\
\hline 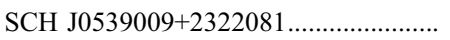 & 19.1 & 17.6 & 15.3 & 12.68 & 12.10 & 11.79 & 2.53 & 1.59 & 0.88 & M6 & -16 & Young \\
\hline
\end{tabular}

Note.-Table 1 is also available in machine-readable form in the electronic edition of the Astronomical Journal.

a Object IDs given in J2000.0 coordinates.

${ }^{\mathrm{b}}$ Near-infrared photometry taken from 2MASS.

c Three of our PMS objects were previously identified in the literature: SCH J0429595+2433080 (Guieu et al. 2006), SCH J0431191+2335048 (Luhman 2006), and SCH J0416272+2053093 (Wichmann et al. 1996).

possibly accreting objects. SCH J0429595+2433080 also has Ca II triplet emission.

\section{NEW PRE-MAIN-SEQUENCE OBJECTS}

From the spectroscopic data we identify 19 objects having spectral features that indicate lower surface gravity than members of USco ( $\sim 5 \mathrm{Myr})$. Most of the new low-gravity objects have inferred gravities as low as those similarly inferred for known 1-2 Myr Taurus members. Of these 19 objects, three were previously identified in the literature: SCH J04295950+2433080 (Guieu et al. 2006), SCH J04311908+2335048 (Luhman 2006), and SCH J0416272+2053093 (Wichmann et al. 1996). We additionally identify 23 objects that have intermediate-strength surface gravity features consistent with those observed in USco,
TW Hya, and $\beta$ Pic stars. In Figure 6 we present spectra of M4/M4.5 stars shown in order of decreasing surface gravity and decreasing $\mathrm{Na}$ I and $\mathrm{K}$ I absorption (bottom to top). All spectra of observed stars as old as AB Dor or the Pleiades ( $100 \mathrm{Myr}$ ) appear identical to those of dwarf stars. Therefore, the intermediate-age population in Taurus is likely significantly younger than $\sim 100 \mathrm{Myr}$, although the exact upper bound on the age of this population is unknown due to a lack of comparison stars with ages between 10 and 100 Myr. This interpretation may also apply to three objects identified by Luhman (2006) that were found to have gravity intermediate between Taurus and dwarf stars and were presumed in that study to be $\sim 100$ Myr old due to the lack of comparison stars with ages between those of Taurus members and field dwarfs. 


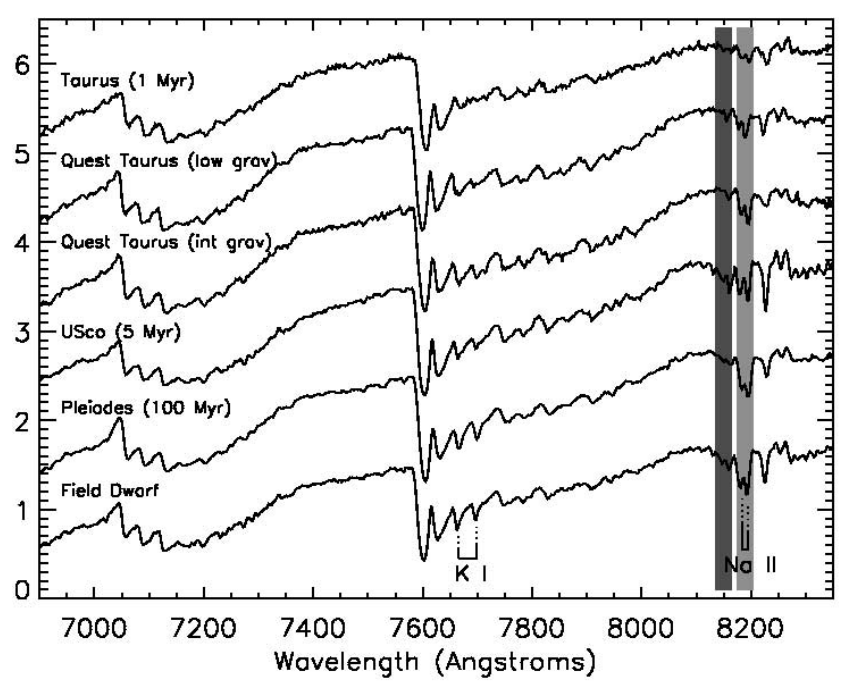

Fig. 6.- Spectra of M4/M4.5 stars presented in order of decreasing surface gravity (bottom to top). Spectra shown are of a field dwarf, a $115 \mathrm{Myr}$ Pleiades object, a 5 Myr USco member, a new Quest-2 Taurus candidate identified to have USco-type intermediate gravity, a new Quest-2 Taurus candidate identified to have low gravity, and a 1 Myr Taurus star. Surface-gravity-sensitive features include the K I doublet $(7677 \AA)$ and the Na I doublet $(8189 \AA)$.

Hereafter we refer to the low-gravity objects as "young" and to the intermediate-gravity objects as "intermediate-age." In the color-magnitude and color-color diagrams (Figs. 1 and 2), candidates for which we have obtained spectral data are shown as large symbols. For both figures, objects confirmed to be young (including SCH J04295950+2433080, SCH J04311908+2335048, and SCH J0416272+2053093) or intermediate-age PMS stars are distinguished from those determined to be older field objects. Photometric and spectral data for new PMS stars is given in Table 1. The Appendix contains magnitudes and spectral indice measurements for PMS candidates spectroscopically determined to be field dwarfs.

Understanding the relationship of the newly identified PMS population to the known Taurus members requires distances to the new PMS stars that we cannot determine based on the current data set. If we assume that they are located at the distance of Taurus, the derived ages from an H-R diagram are 1-10 Myr, and the intermediate-age stars tend to have systematically lower derived luminosities than the young stars at a given spectral type. While these relative ages are consistent with those derived from surface gravity analysis, a range of distances could yield similar luminosity segregation results. In lieu of distance measurements, we constrain the origin of the PMS objects identified in this work by assessing the projected spatial distribution and kinematics of the new young and intermediate-age stars in relation to the known Taurus population.

\subsection{Spatial Distribution}

The new young and intermediate-age stars are distributed throughout the survey region, and many are located well beyond regions previously explored for young PMS stars. Figure 7 shows the location of spectroscopically observed Quest-2 candidates, along with known low-mass Taurus members from the literature (Briceño et al. 2002; Guieu et al. 2006; Hartmann 2002; Luhman et al. 2003; Luhman 2004, 2006). The region that has been previously studied for low mass stars using deep optical/near-infrared imaging with spectroscopic follow-up (Briceño et al. 2002; Guieu et al. 2006; Luhman et al. 2003; Luhman 2000, 2004) is indicated, as is the Pleiades association. Based on comparison with CO maps

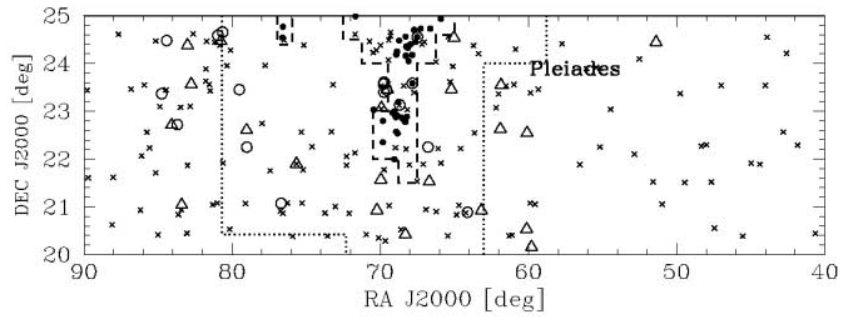

FIG. 7.- Spatial area of the imaging survey shown with previously known low-mass Taurus members ( filled circles; see text for references). Dashed lines indicate the boundary of previous deep CCD imaging surveys aimed at identifying new 1 Myr old association members. Dotted lines indicate the boundary to which a $5 \mathrm{Myr}$ old star with velocity $2 \mathrm{~km} \mathrm{~s}^{-1}$ could have traveled from any of the known subclusters. Open circles and triangles represent new low- and intermediate-gravity stars identified from this work. Black crosses show spectral candidates determined to be field dwarfs. The location of the Pleiades cluster $\left(\alpha=57^{\circ}, \delta=24^{\circ}\right)$ is indicated.

of the region (Dame et al. 2001), while some objects do lie in projection near molecular gas, we see no systematic correlation between the spatial distribution of the new PMS stars and the CO emission.

To assess whether the new PMS stars are associated with the known concentrations of Taurus members or whether they are more uniformly distributed, we show in Figure 8 a histogram of the right ascension values for all sources with spectra presented here and for those sources confirmed as PMS objects. In the middle panel we present the percentage of spectroscopically observed objects determined to be PMS stars. These can be compared to the right ascension range that contains $98 \%$ of the known lowmass Taurus members (Briceño et al. 2002; Guieu et al. 2006;
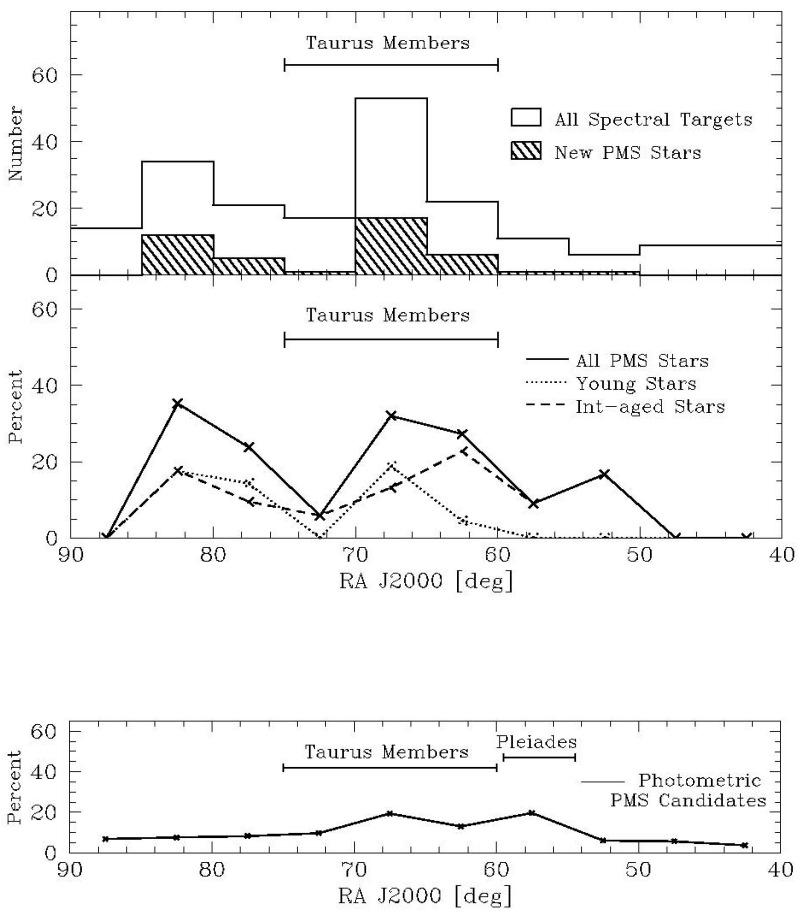

FIG. 8. - Top: Histograms for the total number of stars observed spectroscopically (open histogram) and those determined to be PMS stars (hatched histogram), as a function of right ascension. Middle: Percentage of spectroscopically observed objects classified as PMS stars (solid line) and the percentage determined to be young (dotted line) or intermediate-age (dashed line) stars based on spectroscopic signatures of surface gravity. The right ascension range containing $98 \%$ of known low-mass Taurus members is shown. Bottom: Percentage of the $\sim 1800$ photometric PMS candidates that fall at a given right ascension. 
Hartmann 2002; Luhman et al. 2003; Luhman 2004, 2006). The bottom panel shows the percentage of the 1800 candidates located within a given right ascension range.

We note two spatial concentrations of new PMS stars: one near the known Taurus members at $\alpha \approx 68^{\circ}$ and a second in the eastern portion of the cloud centered around $\alpha \approx 82^{\circ}$. In Figure 7 we indicate the approximate boundary within which a $5 \mathrm{Myr}$ star with velocity $2 \mathrm{~km} \mathrm{~s}^{-1}$ relative to Taurus could have traveled from any of the known Taurus subclusters. Some of the young objects newly identified here are located well beyond this region. Assuming an age of $<5 \mathrm{Myr}$ as derived from surface gravity analysis, if these new young stars originated in the known starforming subclusters they must, therefore, have arrived at their current positions at relatively high velocities.

An alternative and perhaps more likely scenario is that we have identified previously unknown areas of recent star formation outside of the current dense cloud complex. To further quantify the new PMS population and assess this possibility, we divide our PMS stars into three groups: those that lie in the same right ascension range as $98 \%$ of the known Taurus sample $\left(60^{\circ} \leq \alpha \leq\right.$ $\left.75^{\circ}\right)$, those that are east $\left(\alpha>75^{\circ}\right)$ of this right ascension range, and those that are west $\left(\alpha<60^{\circ}\right)$ of this right ascension range. We find that of the 190 spectral candidates, 2 out of $33(6 \%)$ are confirmed to be PMS stars in the western region, 23 out of 89 (26\%) in the central region, and 17 out of $68(25 \%)$ in the eastern region. Spectroscopic confirmation rates of $25 \%$ in the east and only $6 \%$ in the west are contrary to the isotropic distribution we would expect to observe if these stars had been dispersed from the central regions. Using the two-tailed Fisher Exact test we compute a probability of $\sim 3 \%$ that the observed eastern and western distributions could have been drawn from the same population. We can therefore conclude with $97 \%$ confidence that the distributed PMS stars were not randomly dispersed from the known 1-2 Myr old Taurus population. Instead, we suggest that they likely represent a population that is not associated with the currently visible areas of the dense Taurus-Auriga molecular cloud complex.

\subsection{Proper Motions}

A primary goal of our large-area survey is to search for and characterize any PMS stars that might exist far from the $\sim 1-2 \mathrm{Myr}$ old subclusters in Taurus. Having identified several tens of such stars, we can use proper-motion information to further study the characteristics of this spectroscopically selected sample.

We extracted USNO-B1.0 proper motions ${ }^{3}$ (Monet et al. 2003) for 141 of the 190 objects with spectra. Figure 9 shows histograms of the $\alpha$ and $\delta$ proper-motion components for new young and intermediate-age PMS targets and for stars classified as field dwarfs. Because proper motions listed in the USNO catalog are relative rather than absolute (i.e., the proper motions of background stars have not been accounted for), it was necessary to extract similarly derived USNO proper motions of known Taurus members for comparison rather than using more accurate values listed in the literature (e.g., Frink et al. 1997). The bottom panels of Figure 9 show histograms of USNO proper motions for

\footnotetext{
${ }^{3}$ We define an object to have a measured USNO proper motion if it has either a nonzero proper motion or a nonzero proper-motion uncertainty in the USNO-B1.0 Whole Sky catalog. This cut will bias us against objects with in-

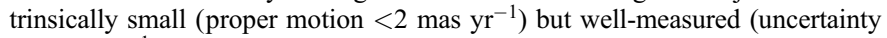

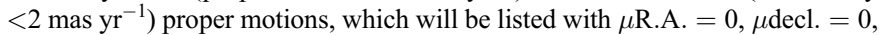
$\mu$ R.A._err $=0$ and $\mu$ decl._err $=0$. However, because the USNO proper-motion measurements are rounded to the nearest mas $\mathrm{yr}^{-1}$ and nonmeasurements are not indicated, we are unsure when a measured proper-motion uncertainty of zero refers to a very small uncertainty vs. a nondetection. Therefore, we feel this is a necessary, if conservative, selection criterion.
}

160 known Taurus members and for 58 Hipparcos-selected Pleiades members (Robichon et al. 1999).

The proper motions of the PMS stars appear strongly correlated (independent of right ascension) and distinct from the proper motions of Pleiades members, whereas the spectroscopic field dwarfs exhibit a very broad distribution of proper motions. These results indicate that the newly identified PMS population is not associated with the Pleiades and is not a collection of random field stars. Further, the PMS stars farthest from the known Taurus population do not exhibit systematically higher proper motions, as would be expected if they had been ejected from the current star-forming regions, giving further evidence that we have identified a new region of relatively recent star formation within the eastern regions of the clouds. The young sample, in particular, appears to have proper motions consistent with Taurus. A possible interpretation of these data is that the new PMS objects in the central regions are associated with the known Taurus population, and the new PMS objects farther away were formed out of molecular material having a velocity and distance similar to that forming the current $1 \mathrm{Myr}$ old population. However, typical USNO proper-motion uncertainties are large $\left( \pm 4\right.$ mas $\left.\mathrm{r}^{-1}\right)$, and the Taurus data itself exhibit a large spread (Fig. 9, bottom panels). Therefore, a more detailed kinematic study of these objects is necessary before a definitive conclusion about their origin can be drawn (see $\S 5$ ).

\subsection{A New Distributed Population and the Post T Tauri Star Problem}

There has been much debate over the last few decades as to whether there exists a population of 3-10 Myr old PTTSs stars associated with the current 1-2 Myr old Taurus members. Spectroscopic follow-up studies of RASS-selected sources have identified a widespread population of stars in the vicinity of Taurus (e.g., Neuhäuser et al. 1997; Wichmann et al. 1996), but the distances, ages, and origins of these stars remain controversial. Measures of X-ray emission and lithium equivalent widths are consistent with any ages from $\approx 1$ to $100 \mathrm{Myr}$ for these objects, and the nearly uniform spatial distribution of this population is consistent with it originating from a number of sources other than Taurus (e.g., Briceño et al. 1997). We have compared the spatial location of the new PMS sources identified here to the distribution of RASS sources in the area (eg., Guillout et al. 1998) and find no correlation between areas of dense X-ray sources detected in the RASS and the newly identified stars in the eastern part of our survey region.

Besides X-ray emission, an efficient method of identifying nearby older PMS stars over a large area is through optical and near-infrared imaging combined with spectroscopic follow-up observations. Surface gravity signatures present in optical spectra of late-K and $\mathrm{M}$ stars offer the advantage of clearly distinguishing young and intermediate-age PMS stars from 75-100 Myr old stars. Considering the large number of imaging/spectroscopic surveys in the Taurus region, a natural question to ask is: how were the PMS stars presented here not discovered prior to our survey?

The primary reason these objects were not identified in earlier work is that we have searched much farther away from the clouds than most previous photometric/spectroscopic studies. The largearea work by Luhman (2006) probed only as far east as $\alpha \approx 5 \mathrm{hr}$ and did not extend to the large number of newly discovered PMS stars in the eastern portion of our survey at $\alpha>80^{\circ}$. In addition, our survey occupies a unique range in color/magnitude space. Low-mass stars discovered in early CCD surveys (e.g., Briceño et al. 2002) are saturated in the Quest-2 data. More recent surveys (e.g., Luhman 2004, 2006; Guieu et al. 2006) have been concerned 

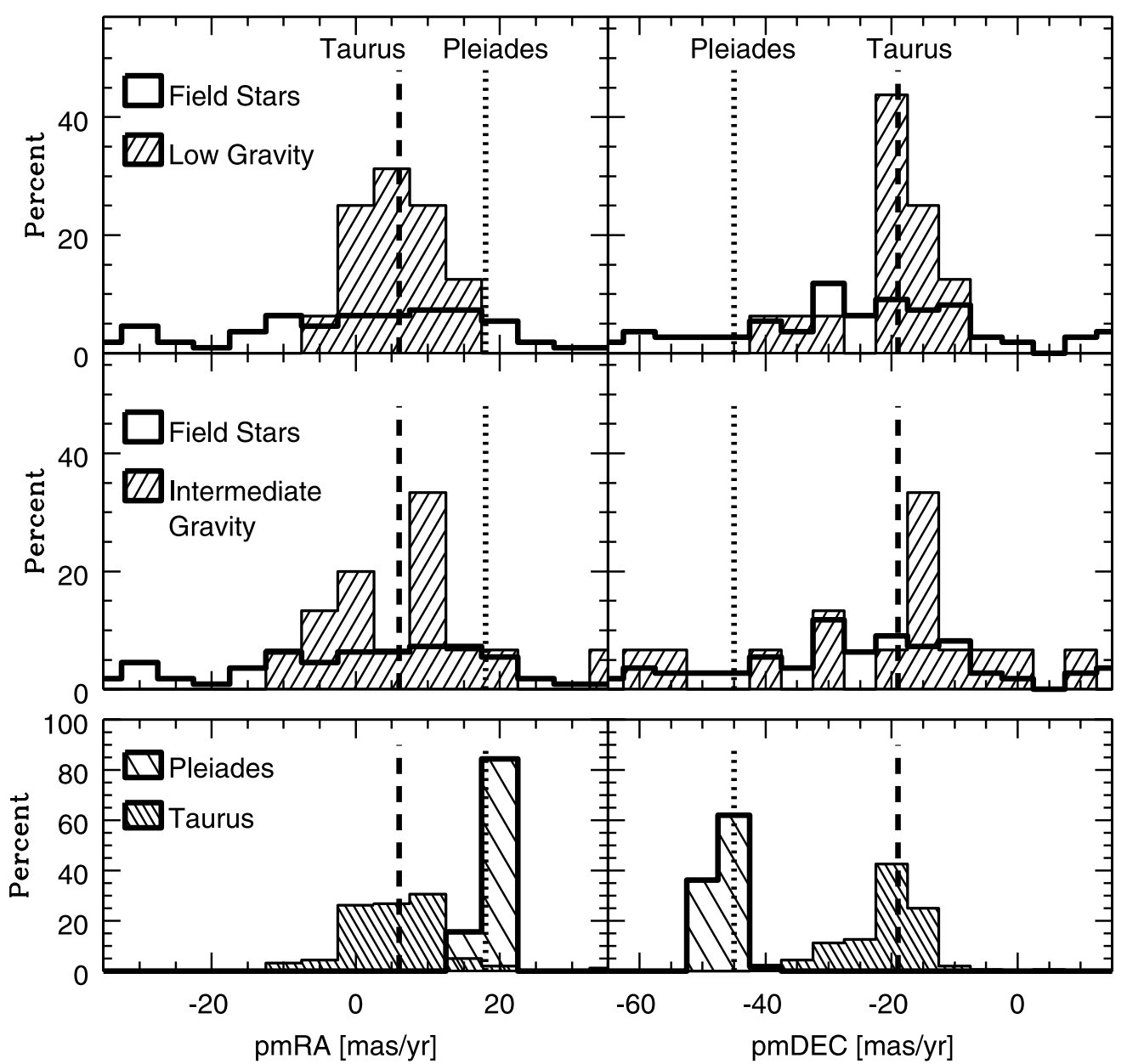

FIG. 9.- - Histograms of proper motions extracted from the USNO-B1.0 catalog. In the top and middle panels, open histograms represent proper motions for stars that were spectroscopically determined to be field dwarfs; hatched histograms represent proper motions for new young (top) and intermediate-age (middle) PMS targets. In the bottom panels, the light hatched histogram shows USNO-B1.0 proper motions for a sample of Pleiades members; the dark hatched histogram shows equivalent data for the sample of known Taurus members indicated in Fig. 7. All histograms have been normalized for ease of comparison, and average values for Taurus and the Pleiades are

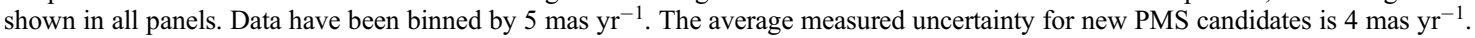

primarily with finding new brown dwarfs with spectral types $\geq$ M6, which will be predominantly fainter and redder than the candidates discussed here. Indeed, comparison of USNO I2 and 2MASS $J, H, K_{s}$ photometry for the Quest-2 PMS candidates to those selected in the Luhman (2006) study reveals that the Quest-2 candidates are systematically bluer. Despite the large overlap in area $\left(\sim 50 \mathrm{deg}^{2}\right)$, the only observed candidate in our survey with spectral type $\geq \mathrm{M} 6$ selected as a candidate via the Luhman (2006) criteria is the one star (SCH J04311908+ 2335048) that our two works both identified. It is unlikely that the intermediate-age objects found in this work, which are predominantly bluer than the young objects (see Fig. 1), would have been selected as candidate Taurus members in any of the previous optical studies.

While we have discovered a new distributed population of both young and intermediate-age stars, we do not at this time claim that these objects represent the PTTSs in Taurus. The spatial distribution of the PMS stars identified far from the known members is not consistent with those stars being associated with the Taurus subclusters. In addition, the fact that the proper motions for the new young stars located tens of degrees away are consistent with known Taurus members implies that they were not ejected at high velocities from the current star-forming regions. Because our data set is not complete either spatially or in magnitude/color space (due to difficulties with weather and calibration), we cannot assess the full extent of this new population. Rather, we note that its existence hints there may be many more as-yet undiscovered PMS objects waiting to be identified in and surrounding the Taurus clouds.

\section{SUMMARY AND FUTURE WORK}

We have completed a large-area $\left(\sim 250 \mathrm{deg}^{2}\right) B, R, I$ imaging survey of the southern part of the Taurus molecular cloud using the Quest-2 camera. From a combination of these data and 2MASS $J, H, K_{s}$ photometry, candidate PMS objects were selected throughout the region. We have observed spectra of 190 of these objects $(\approx 10 \%$ of the photometrically selected candidates) and, based on comparison to spectra of Taurus members and members of other young associations, have determined that 42 are bona fide PMS stars. From the strength of spectral features sensitive to surface gravity, we subdivide the new PMS objects into young and intermediate-age stars. We find members of both populations throughout the survey region and well beyond previously studied areas. In particular, we find a new concentration of PMS objects in the eastern portion of the cloud located at $\alpha \sim 82^{\circ}$ and $\delta \sim$ $24^{\circ}$, which likely did not originate from the previously known starforming regions. We have analyzed the USNO proper motions of 141 spectral targets and find a strong concentration of proper motions for the new PMS stars around those measured for known Taurus members. Conversely, the spectroscopic field dwarf population exhibits a very broad distribution of proper motions. 
TABLE A1

Measured Quantities for Candidates Spectroscopically Confirmed as Field Dwarfs

\begin{tabular}{|c|c|c|c|c|c|c|c|c|c|}
\hline $\mathrm{ID}^{\mathrm{a}}$ & $b$ & $r$ & $i$ & $J^{\mathrm{b}}$ & $H^{\mathrm{b}}$ & $K_{S}^{\mathrm{b}}$ & TiO-7140 & TiO-8165 & $\mathrm{Na}-8195$ \\
\hline SCH J02423426+20262686 $\ldots \ldots \ldots \ldots \ldots \ldots$ & 17.3 & $\ldots$ & 14.4 & 12.53 & 11.98 & 11.69 & 1.55 & 1.07 & 0.87 \\
\hline SCH J02471836+22171512 …................ & $\ldots$ & 20.2 & 17.9 & 14.58 & 13.94 & 13.49 & 3.17 & 1.85 & 0.76 \\
\hline SCH J02502040+24124969................... & 18.4 & 16.5 & 14.9 & 12.55 & 11.94 & 11.63 & 1.98 & 1.22 & 0.81 \\
\hline SCH J02511084+22335835 ................... & 17.8 & 16.1 & 14.7 & 12.52 & 11.90 & 11.66 & 1.84 & 1.16 & 0.86 \\
\hline 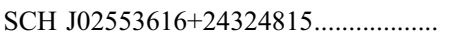 & 19.2 & 17.3 & 15.5 & 12.82 & 12.18 & 11.83 & 2.28 & 1.35 & 0.77 \\
\hline SCH J02560113+23322159................... & 19.2 & 17.5 & 15.4 & 13.13 & 12.55 & 12.21 & 2.11 & 1.26 & 0.78 \\
\hline SCH J02573373+21531119 _.................. & 17.0 & 15.4 & 13.9 & 11.58 & 11.03 & 10.74 & 1.84 & 1.14 & 0.84 \\
\hline SCH J02595189+21544806................... & 18.8 & 17.1 & 15.5 & 13.00 & 12.36 & 12.07 & 2.03 & 1.25 & 0.82 \\
\hline 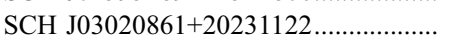 & 17.3 & 15.7 & 14.0 & 11.68 & 11.05 & 10.79 & 2.06 & 1.25 & 0.88 \\
\hline SCH J03075903+23322049.................. & 17.6 & 16.0 & 14.0 & 12.31 & 11.74 & 11.46 & 1.71 & 1.10 & 0.84 \\
\hline
\end{tabular}

NoтеS.-Table A1 is published in its entirety in the electronic edition of the Astronomical Journal. A portion is shown here for guidance regarding its form and content.

a Object IDs given in J2000.0 coordinates.

b Two faint candidates observed during the first spectroscopic observing run before the final photometric calibrations were finished do not have $r$-band photometry.

${ }^{\mathrm{c}}$ Near-infrared photometry taken from 2MASS.

Radial velocities from high-resolution spectra for all 42 new PMS stars combined with more robust proper-motion measurements derived from existing catalogs will allow us to compute a three-dimensional $U, V, W$ velocity for each object over a range of distances. In addition, lithium equivalent widths can help confirm the youth of the new PMS sample, which is currently based on $\mathrm{Na}$ I and $\mathrm{K}$ I line strengths. While lithium depletion occurs slowly over $>100$ Myr for G-type stars and is therefore not suitable for distinguishing PTTSs from $100 \mathrm{Myr}$ objects at these temperatures, for $\mathrm{K}$ to mid-M type stars that are fully convective lithium depletion occurs over much faster timescales ( 20 Myr; e.g., D'Antona \& Mazzitelli 1994) and is a more robust indicator of youth. Finally, we emphasize that the new PMS population of 42 stars and a spectroscopic confirmation rate of $\sim 20 \%$ implies that several hundred similar young and intermediate-age PMS stars may be present in our larger photometric database.
The authors are appreciative of the Quest-2 collaboration, including David Rabinowitz, Anne Bauer, and Jonathan Jerke, for observing and processing the photometric drift-scan data. We would like to thank Ashish Mahabal and Eilat Glikman for many discussions concerning the systematics and calibration of the Quest-2 data. We thank Lee Hartmann for his insights and suggestions, which improved the quality of this manuscript. We are grateful to assistance from the entire Palomar staff, in particular Jean Mueller, Karl Dunscombe, and Dipali. This manuscript has made use of data from the Two Micron All Sky Survey.

\section{APPENDIX}

\section{MEASURED QUANTITIES FOR FIELD DWARFS}

Photometry and spectral indice measurements of the candidates determined to be field stars are listed in Table A1.
Ardila, D., Martín, E. L., \& Basri, G. 2000, AJ, 120, 479

Basri, G., Marcy, G., \& Graham, J. 1996, ApJ, 458, 600

Blitz, L., \& Shu, F. H. 1980, ApJ, 238, 148

Briceño, C., Calvet, N., Kenyon, S., \& Hartmann, L. 1999, AJ, 118, 1354

Briceño, C., Hartmann, L., Stauffer, J., \&Martín, E. 1998, AJ, 115, 2074

Briceño, C., Luhman, K. L., Hartmann, L., Stauffer, J. R., \& Kirkpatrick, D. 2002, ApJ, 580, 317

Briceño, C., et al. 1997, AJ, 113, 740

Carpenter, J. M. 2000, AJ, 120, 3139

Dame, T. M., Hartmann, D., Thaddeus, P. 2001, ApJ, 547, 792

D’Antona, F., \& Mazzitelli, I. 1994, ApJS, 90, 467

de la Reza, R., Jilinski, E., \& Ortega, V. G. 2006, AJ, 131, 2609

Frink, S., Neuhäuser, R., \& Sterzik, M. F. 1997, A\&A, 325, 613

Gomez, M., Hartmann, L., Kenyon, S. J., \& Hewett, R. 1993, AJ, 105, 1927

Guieu, S., Dougados, C., Monin, J.-L., Magnier, E., \& Martín, E. L. 2006, A\&A, 446, 485

Guillout, P., Sterzik, M. F., Schmitt, J. H. M. M, Motch, C., \& Neuhäuser, R. 1998, A\&A, 337, 113

Hartmann, L. 2000, in Star Formation from the Small to Large Scale, ed. F.

Favata, A. Kaas, \& A. Wilson (ESA SP-445; Noordwijk: ESA), 107

- 2002, ApJ, 578, 914

Hartmann, L., Ballesteros-Paredes, J., \& Bergin, E. 2001, ApJ, 562, 852

Hartmann, L., Hewett, R., Stahler, S., \& Mathieu, R. D. 1986, ApJ, 309, 275

Herbig, G. H. 1978, in Problems of Physics and Evolution of the Universe, ed. L. V. Mirzoyan (Yerevan: Armenian Acad. Sci.), 171

\section{REFERENCES}

Kenyon, S., Dobrzycka, D., \& Hartmann, L. 1994, AJ, 108, 1872

Kirkpatrick, J. D., Henry, T. J., \& McCarthy, D. W. 1991, ApJS, 77, 417

Lebreton, Y., Fernandes, J., \& Lejeune, T. 2001, A\&A, 374, 540

Luhman, K. L. 2000, ApJ, 544, 1044

-.2004, ApJ, 617, 1216

- 2006, ApJ, 645, 676

Luhman, K. L., Briceño, C., Stauffer, J. R., Hartmann, L., Barrado Y Navascués, D., \& Caldwell, N 2003, ApJ, 590, 348

Luhman, K. L., Stauffer, J. R., \& Mamajek, E. E. 2005, ApJ, 628, L69

Massey, P., Strobel, K., Barnes, J., Anderson, E. 1988, ApJ, 328, 315

Monet, D. G., et al. 2003, AJ, 125, 984

Neuhäuser, R., Torres, G., Sterzik, M. F., \& Randich, S. 1997, A\&A, 325, 647

Ortega, V. G., de la Reza, R., Jilinski, E., \& Bazzanella, B. 2004, ApJ, 609, 243

Palla, F., \& Stahler, S. W. 2000, ApJ, 540, 255

Rabinowitz, D., et al. 2003, BAAS, 35, 1262

Rebull, L. M., et al. 2000, AJ, 119, 3026

Robichon, N., Arenou, F., Mermilliod, J.-C., \& Turon, C. 1999, A\&A, 345, 471

Schlegel, D. J., Finkbeiner, D. P., \& Davis, M. 1998, ApJ, 500, 525

Skrutskie, M. F., et al. 2006, AJ, 131, 1163

Slesnick, C. L., Carpenter, J. M., \& Hillenbrand, L. A. 2006, AJ, 131, 3016 (SCH06)

Wichmann, R., et al. 1996, A\&A, 312, 439

Wilking, B. A., Meyer, M. R., Robinson, J. G., \& Greene, T. P. 2005, AJ, 130, 1733

York, D. G., et al. 2000, AJ, 120, 1579 